\title{
The Nasogastric Tube in the Thorax after Gastric Pull Up!
}

\author{
Puneet Khanna, Rakesh Garg, Amit Chirom Singh
}

Department of Anaesthesiology and Intensive Care, All India Institute of Medical Sciences, New Delhi, India.

Email: drrgarg@hotmail.com

Received December $18^{\text {th }}, 2011$; revised January $4^{\text {th }}, 2012$; accepted January $15^{\text {th }}, 2012$

\begin{abstract}
Although nasogastric tube (NGT) is a simple procedure, the blind placement of nasogastric feeding tubes is not without risks. Chest radiogram is done to confirm the correct position of the NGT. We report a case where radiography could not be confirmatory for the correct position of the tube in a specific group of patients.
\end{abstract}

Keywords: Nasogastric Tube; Misplaced; Esophagectomy

\section{Introduction}

Nasogastric tube (NGT) is inserted for providing nutriation, drugs or for gastric drainage. Although a simple procedure, the blind placement of nasogastric feeding tubes is not without risks. There could be misplacement during insertion or may perforate the esophagus or stomach [1]. More than 50\% of malpositioned blindly inserted nasogastric feeding tubes occur in patients with endotracheal tube or tracheostomy [2]. The various routine methods for confirming the position of the NGT includes auscultation of air insufflated through the feeding tube (whoosh test), testing acidity/alkalinity of the aspirate, interpreting absence of respiratory distress as an indicator of correct positioning, monitoring bubbling at the end of NGT and observing the appearance of the feeding tube aspirate. But these methods are not reliable [3]. The reliable methods include measuring the $\mathrm{pH}$ of the aspirate and use of radiography which is considered to be most confirmatory [3]. We report a case where radiography could not be confirmatory for the correct position of the tube in a specific group of patients.

\section{Case}

A 22-year-old male patient was scheduled for tracheal dilation under general anaesthesia. He had a history of acid ingestion leading to stricture esophagus and tracheal stenosis. He underwent tracheostomy and esophagectomy with gastric pull up. Presently, anesthesia was induced with intravenous fentanyl $100 \mu \mathrm{g}$ and propofol 100 mg and muscle relaxation was achieved by atracurium 30 mg and lungs were ventilated via tracheostomy tube. After induction of anaesthesia nasogastric tube was placed for gastric drainage. On auscultation at the epigastric area, air sound could not be heard. On laryngo- scopy, the NGT tube appeared to enter the esophageal opening. Our concern for misplaced NGT included any perforation injury. A chest X-ray (AP, lateral view) was done and NGT was found to be coiled in the thoracic area and tip was present in the anterior mediastinal area (Figure 1, 2). Patient was haemodyanamically stable and did not have any respiratory compromise. The $\mathrm{pH}$ of the aspirate confirmed its gastric placement.

\section{Discussion}

A nasogastric feeding tube is considered malpositioned if it does not stay within the esophageal lumen or if the distal end of the tube is not below the gastroesophageal

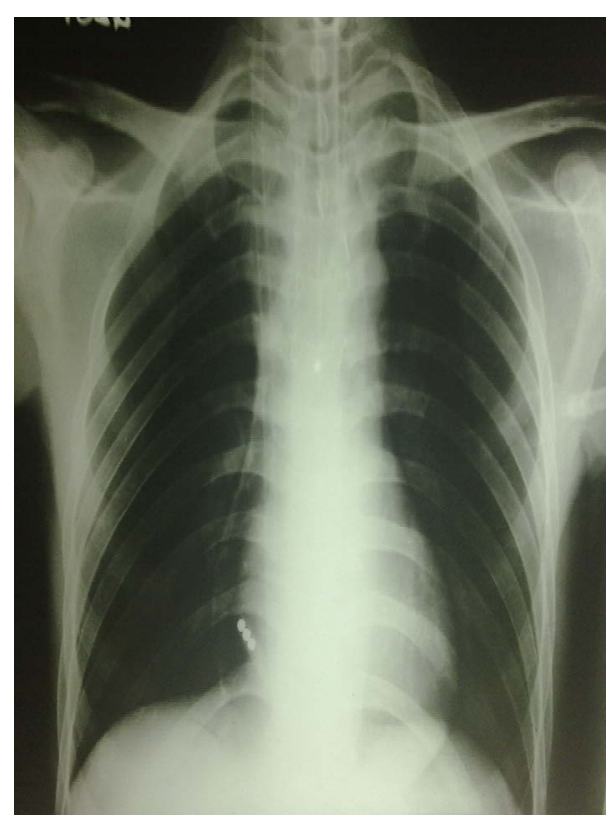

Figure 1. Chest X-ray AP view. 


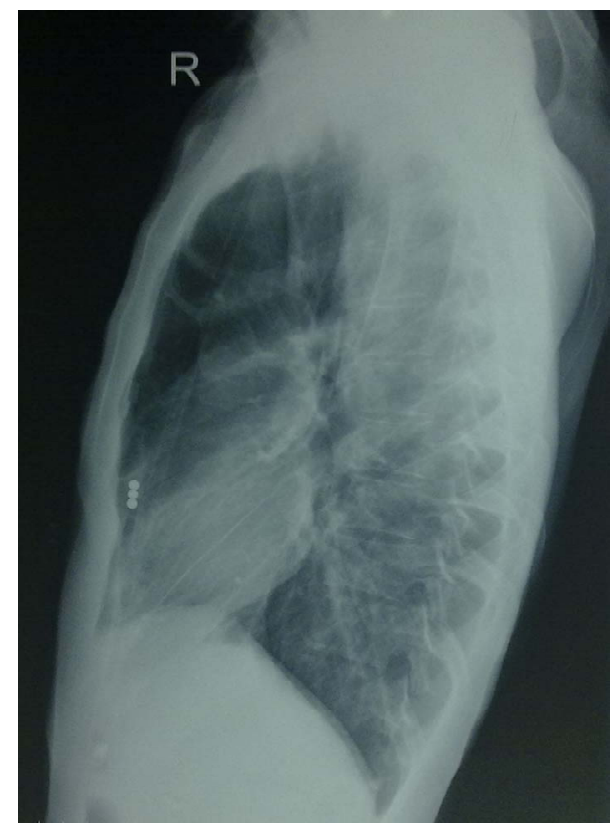

Figure 2. Chest X-ray lateral view.

junction. But in patients with esophagectomy and gastric pull up, the optimal position of the NGT tip and its confirmatory method is not mentioned in the literature. Single method of detecting the correct placement of the NGT may miss the misplaced NGT in patients who have altered anatomy due to surgical procedure. We suggest in patients with esophagectomy and gastric pull up, the confirmation of the correct placement of the NGT should be done by more than one methods including history of previous surgical procedure, $\mathrm{pH}$ of the aspirate and the radiographic evaluation. Also the NGT tube should be placed under direct vision using the laryngoscope and we should be cautious in interpreting the chest radiograph for correct placement of NGT in patient with gastric pull up to avoid any missed incidences of perforation injuries. In case NGT is required for a long term, then it will be advisable to introduce NGT under endoscopic or fluoroscopic guidance for its correct placement [4-6].

We conclude that placement of NGT in patients after esophageal surgical procedure should be confirmed using multiple methods including endoscopic visualization for its optimal visualization rather than conventional technique for positioning of the NGT.

\section{REFERENCES}

[1] J. Tait, "Going Nasogastric: Current Thinking in Nasogastric Tube Techniques,” Complete Nutrition, Vol. 1, No. 2, 2001, pp. 27-29.

[2] R. Sorokin and J. E. Gottlieb, "Enhancing Patient Safety during Feeding Tube Insertion: A Review of More than 2000 Insertions," Journal of Parenteral and Enteral Nutrition, Vol. 30, No. 5, 2006, pp. 440-445. doi:10.1177/0148607106030005440

[3] L. J. E. Walker, "Methods to Correct Placement of a Nnasogastric Tube: Beware of Pitfalls,” Age and Ageing, Vol. 34, No. 6, 2005, pp. 655-657. doi:10.1093/ageing/afi177

[4] N. A, Metheny and K. L. Meert, "Monitoring Feeding Ttube Placement," Nutrition in Clinical Practice, Vol. 19, No. 5, 2004, pp. 487-495. doi:10.1177/0115426504019005487

[5] S. Huffman, et al., "Methods for Confirming Feeding Tube Placement: Application of Research in Practice," Peadiatric Nursing, Vol. 30, No. 1, 2004, pp. 10-12.

[6] S. Tamhene, D. Tuthill and A. Evans, "Should Ultrasound Be Routinely Used to Confirm Correct Positioning of Nasogastric Tubes in Neonates?” Archives of Disease in Childhood Fetal and Neonatal Edition, Vol. 91, 2006, p. 388. doi:10.1136/adc. 2005.088476 